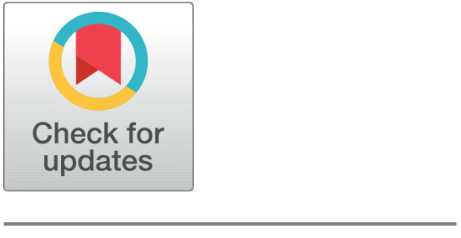

OPEN ACCESS

Received: 20.10 .2021

Accepted: 27.11 .2021

Published: 28.12 .2021

Citation: Achar SJ, Baishya C (2021) Mathematical Analysis of

Pluviculture in the Frame of Caputo Fractional Derivative. Indian Journal of Science and Technology 14(48): 3509-3524. https://doi.org/ 10.17485/IJST/v14i48.1965

* Corresponding author.

sindhuachar6@gmail.com

Funding: None

Competing Interests: None

Copyright: (c) 2021 Achar \& Baishya. This is an open access article distributed under the terms of the Creative Commons Attribution License, which permits unrestricted use, distribution, and reproduction in any medium, provided the original author and source are credited.

Published By Indian Society for Education and Environment (iSee)

ISSN

Print: 0974-6846

Electronic: 0974-5645

\section{Mathematical Analysis of Pluviculture in the Frame of Caputo Fractional Derivative}

\author{
Sindhu J Achar ${ }^{1 *}$, Chandrali Baishya ${ }^{1}$ \\ 1 Department of Studies and Research in Mathematics, Tumkur University, Tumkur-572103, \\ Karnataka, India
}

\section{Abstract}

Objectives: Climate change is a major issue the mankind is facing in the present scenario. This is a consequence of global warming that result in the change in temperature and rainfall over a long period of time. In this work, we analyze the fractional mathematical model projecting pluviculture. The Caputo fractional derivative is incorporated for better analysis of this event. Also, we discuss the boundedness, existence and uniqueness of the solutions of the proposed system. Sufficient conditions required for existence of asymptotic stability are discussed. Method: We have used the generalized AdamsBashforth-Moulton predictor-corrector technique to solve the pluviculture model. It is the linear multistep implicit method used to solve the system of equations. Findings: It is established that, the intensity of precipitation is enhanced by introducing the aerosols to the water vapor. The incorporation of the fractional derivatives strengthens the model in a more realistic way. The influence of some parameters and fractional derivative on the rain making process are numerically analyzed. Novelty: The incorporation of Caputo fractional derivative to the pluviculture model along with the two kinds of aerosols is the novel in the model.

Keywords: Pluviculture; Aerosol; Caputo fractional derivative;

AdamsBashforthMoulton technique Mathematics Subject Classification:

26A33; 92D30

\section{Introduction}

Rainmaking, also known as pluviculture, is the method of inducing precipitation artificially. This is done to stave off drought and to save Earth from wider global warming. This can be achieved using rockets or airplanes to sow to the clouds along with the catalysts like silver iodide, ${ }^{(1)}$ dry ice and salt powder, to increase precipitation and mitigate farmland drought. The concept of "pluviculture," or the artificial rain creations, has long history, both ancient and modern. Especially during the 19th and 20th centuries, it has excited the mind's eye and brought forth enormous rainmaking techniques. Rain making is a prime and complex phenomenon in the environment. Several physicochemical processes such as agglomeration, nucleation, condensation and so on are involved in this process. ${ }^{(2,3)}$ Firstly, water vapors are converted to cloud 
droplets of small size by condensation and nucleation processes. Then, the small droplets randomly move inside the cloud, stick and collide to each other and forms large size cloud drops. Further, the cloud droplets of large size are converted into rain droplets by the process of agglomeration and nucleation. Aerosols has the vital role in this process which in turn enhances the precipitation. Aerosols refer to the tiny specks which are in solid or liquid state having the diameters in the range $10^{-9}$ to $10^{-4} \mathrm{~m}$. They originate from anthropogenic springs and also created in the nature by transformation of gas into particles due to several chemical reactions. The key components of aerosols are sodium chloride, gritty particles, nitrate, crustal elements and biogenic organic molecules like spores, fragments of the plant and pollen.

Some theoretical and as laboratory experiments are carried out and seen that aerosols play the role of Cloud Condensation Nuclei (CCN), surrounding to which the cloud droplets generates. ${ }^{(4-8)}$ Some studies show the analysis of the deletion of dusty particles of gaseous state from environment by bearing the synergy of these particles with the cloud drops. It is notable from the above studies that, the rain and wind play the major role in deletion of pollutants present in the local regional environment. ${ }^{(9-11)}$ Some studies show the role of aerosols in the regional environment for inducing artificial rain. ${ }^{(12-14)}$ In particular, Misra et al. ${ }^{(15)}$ proposed the mathematical model for the artificial precipitation by presuming that two types of aerosols are instigated to environment where water vapors generates in an uninterrupted manner. Aerosols of first kind when associated with water vapors converts to cloud drops. The aerosols of second kind collide with the cloud drops and transform them to raindrops which finally leads to precipitation.

The development and use of fractional calculus has proven to be an useful tool. The Caputo, Gr ̈̈ nwald Letnikov, Riemann-Liouville, Atangana-Baleanu, Jumarie, Caputo-Fabrizio are few among the fractional derivatives that are gaining their importance among researchers to replicate real world problems. Over the years, theories of these derivatives have been developed to a great extent. ${ }^{(16,17)}$ Applications of Caputo-Fabrizio fractional and Atangana-Baleanu derivative can be found in. ${ }^{(18-23)}$ Many phenomena of mathematical biology ${ }^{(24-26)}$ and their interdisciplinary fields ${ }^{(27-30)}$ have been studied in a better way by using these fractional derivatives.

From above, it may be pointed out that chemical and physical properties of the natural atmospheric aerosols are much studied but the study of the use of aerosols in making precipitation is given a little attention. Especially the combination of two kinds of drops (big and small) and two kinds of aerosols is given even less attention. Also, the pluviculture model with the fractional order is nowhere discussed. Hence, keeping all these in view, in the present work, we have proposed a mathematical model including the two kinds of aerosols in the atmosphere leading to the precipitation incorporating the Caputo fractional derivative which is the novel in the model. In the modeling procedure, we have assumed that the water vapors are formed naturally in the air. But, in order to result in the precipitation, they are not condensed in the required proportion so as to form the cloud droplets which is necessary for rainfall. By introducing the combination of two kinds of aerosols in the atmosphere, the cloud droplets are formed which are of different sizes from the water vapor through the processes of agglomeration, nucleation, and condensation and so on, consequently these changes to water drops which finally leads to precipitation.

\section{Some Essential Theorems}

In the present work, we have used the Caputo fractional derivatives as it supports the integer order initial condition. We have also gone over certain theorems which is utilised to ascertain the stability of equilibrium locations. The Caputo fractional derivative is denoted by a capital letter with an upper-left index ${ }^{C} D$.

Definition 2.1 ${ }^{(16)}$ (Caputo Fractional Derivative) Suppose $g(t)$ is $k$ times continuously differentiable function and $g^{(k)}(t)$ is integrable in $\left[t_{0}, T\right]$. For $g(t)$, The fractional derivative of the order $\alpha$ established by Caputo sense is

$$
{ }_{t_{0}}^{C} D_{t}^{\alpha} g(t)=\frac{1}{\Gamma(k-\alpha)} \int_{t_{0}}^{t} \frac{g^{(k)}(\tau)}{(t-\tau)^{\alpha+1-k}} d \tau
$$

where $\Gamma$ (.) refers to Gamma function, $t>a$ and $k$ is the positive integer with the property that $k-1<\alpha<k$.

Lemma $1^{(31)}$ Consider the system

$$
{ }_{t_{0}}^{C} D_{t}^{\alpha} v(t)=g(t, v), t>t_{0}
$$

choosing the initial condition as $v\left(t_{0}\right)$, where $0<\alpha \leq 1$ and $g:\left(t_{0}, \infty\right) \times \Omega \rightarrow R^{n}, \Omega \in R^{n}$. When $g(t, v)$ holds the locally Lipchitz conditions concerning to $v$, Eq. 1 has a unique solution on $\left(t_{0}, \infty\right) \times \Omega$.

Lemma $2{ }^{(29)}$ We assume that $g(t)$ is the continuous function on $\left(t_{0}, \infty\right)$ satisfying

$$
{ }_{t_{0}}^{C} D_{t}^{\alpha} g(t) \leq-\lambda g(t)+\xi, g\left(t_{0}\right)=f_{t_{0}}
$$


here $0<\alpha \leq 1,(\lambda, \xi) \in R^{2}$ and $\lambda \neq 0$. Consider $t_{0} \geq 0$ as the initial time. Now,

$$
g(t) \leq\left(g\left(t_{0}\right)-\frac{\xi}{\lambda}\right) E_{\alpha}-\left[\lambda\left(t-t_{0}\right)^{\alpha}\right]+\frac{\xi}{\lambda}
$$

\section{Model Formulation}

Motivated by the mathematical models concerning the artificial precipitation process, here, we have analyzed the fractional pluviculture model with the aid of the Caputo fractional order derivative. The pluviculture model is ruled by six interacting variables viz. densities of water vapor $\left(C_{V}\right)$, densities of small size cloud droplets $\left(C_{S D}\right)$, densities of large size cloud droplets $\left(C_{L D}\right)$, densities of rain drops $\left(C_{R}\right)$ and concentrations of the first and second type aerosols $C_{1}$ and $C_{2}$ respectively.

$$
\begin{aligned}
& { }_{t_{0}} D_{t}^{\alpha} C_{V}=J_{V}-\beta_{0} C_{V}-\beta_{1} C_{V} C_{1} \\
& \stackrel{C}{t_{0}} D_{t}^{\alpha} C_{S D}=\delta_{S} C_{V}-\delta_{S_{0}} C_{S D}+\psi \beta_{1} C_{V} C_{1}-\delta_{S_{1}} C_{S D} C_{2} \\
& \stackrel{C}{t_{0}} D_{t}^{\alpha} C_{L D}=\delta_{L} C_{S D}-\delta_{L_{0}} C_{L D}+\delta_{S_{1}} C_{S D} C_{2}-\delta_{L_{1}} C_{L D} C_{2} \\
& \stackrel{c}{t_{0}} D_{t}^{\alpha} C_{R}=\gamma C_{L D}-\gamma_{0} C_{R}+\xi \delta_{L_{1}} C_{L D} C_{2} \\
& \stackrel{0}{t_{0}} D_{t}^{\alpha} C_{1}=J_{1}-\mu_{1} C_{1}-\beta_{1} C_{V} C_{1} \\
& \stackrel{C}{t_{0}} D_{t}^{\alpha} C_{2}=J_{2}-\mu_{2} C_{2}-\delta_{S_{1}} C_{S D} C_{2}-\delta_{L_{1}} C_{L D} C_{2}-\delta_{R} C_{R} C_{2}
\end{aligned}
$$

with initial condition $C_{V}\left(t_{0}\right)>0, C_{S D}\left(t_{0}\right)>0, C_{L D}\left(t_{0}\right)>0, C_{R}\left(t_{0}\right)>0, C_{1}\left(t_{0}\right)>0, C_{2}\left(t_{0}\right)>0$ where $t_{0}$ is the initial time. All the parameters $J_{V}, \beta_{0}, \beta_{1}, \delta_{S}, \delta_{S_{0}}, \psi, \delta_{S_{1}}, \delta_{L}, \delta_{L_{0}}, \delta_{L_{1}}, \gamma, \gamma_{0}, \xi, J_{1}, J_{2}, \mu_{1}, \mu_{2}, \delta_{R}$ are non-negative.

In the projected model 2, the phase of the water vapors are generated in the continuous basis at the rate $J_{V}$ (the net rate of change in the density of the water vapor is presumed to be a constant). The conductive aerosols of the first kind and the second kind are introduced continuously to the environment at the rates $J_{1}$ and $J_{2}$ respectively. The constant terms $\beta_{0}, \delta_{S_{0}}, \delta_{L_{0}}, \gamma_{0}, \mu_{1}$ and $\mu_{2}$ denote the coefficients of natural reduction rate of $C_{V}, C_{S D}, C_{L D}, C_{R}, C_{1}$ and $C_{2}$ respectively.

The coefficients $\delta_{S} \geq 0, \delta_{L}>0$ and $r>0$ denote the rate of natural genesis of small size cloud drops from the water vapor, cloud drops of large size from cloud drops of small size and then to rain drops from the cloud drops of large size. Hence, clearly it can be noted that $\delta_{S} \leq \beta_{0}, \delta_{L}<\delta_{S_{0}}$ and $r<\delta_{L_{0}}$. The terms $\psi$ and $\xi$ are the positive proportionality constants. The coefficients $\beta_{1}, \delta_{S_{1}}, \delta_{L_{1}}$ and $\delta_{R}$ denote the rate of conversion between the water vapor phase and first kind aerosol, cloud drops of small size and second kind aerosol, cloud drops of large size and second kind aerosol and rain drops and the second kind aerosol respectively.

\section{Existence of the solutions}

The existence of the solutions for the pluviculture model 2 is demonstrated using the Fixed-Point Theorem in this Section. There are no precise algorithms or approaches for evaluating the exact solutions since the model is complex and non-local. However, the existence is guaranteed if certain conditions are met. The system 2 can be rewritten as:

$$
\begin{aligned}
& { }_{t_{0}}^{C} D_{t}^{\alpha}\left[C_{V}(t)\right]=\mathrm{P}_{1}\left(t, C_{V}\right) \\
& { }_{t_{0}}^{C} D_{t}^{\alpha}\left[C_{S D}(t)\right]=\mathrm{P}_{2}\left(t, C_{S D}\right) \\
& { }_{t_{0}}^{C} D_{t}^{\alpha}\left[C_{L D}(t)\right]=P_{3}\left(t, C_{L D}\right) \\
& { }^{C} D_{t}^{\alpha}\left[C_{R}(t)\right]=\mathrm{P}_{4}\left(t, C_{R}\right) \\
& { }_{t_{0}}^{C} D_{t}^{\alpha}\left[C_{1}(t)\right]=\mathrm{P}_{5}\left(t, C_{1}\right) \\
& { }_{t_{0}}^{C} D_{t}^{\alpha}\left[C_{2}(t)\right]=\mathrm{P}_{6}\left(t, C_{2}\right)
\end{aligned}
$$

he above system can be transformed into Volterra type integral equation as:

$$
\begin{gathered}
C_{V}(t)-C_{V}(0)=\frac{1}{\Gamma(\alpha)} \int_{0}^{t} P_{1}\left(\tau, C_{V}(\tau)\right)(t-\tau)^{\alpha-1} d \tau \\
C_{S D}(t)-C_{S D}(0)=\frac{1}{\Gamma(\alpha)} \int_{0}^{t} P_{2}\left(\tau, C_{S D}(\tau)\right)(t-\tau)^{\alpha-1} d \tau
\end{gathered}
$$




$$
\begin{gathered}
C_{L D}(t)-C_{L D}(0)=\frac{1}{\Gamma(\alpha)} \int_{0}^{t} P_{3}\left(\tau, C_{L D}(\tau)\right)(t-\tau)^{\alpha-1} d \tau \\
C_{R}(t)-C_{R}(0)=\frac{1}{\Gamma(\alpha)} \int_{0}^{t} P_{4}\left(\tau, C_{R}(\tau)\right)(t-\tau)^{\alpha-1} d \tau \\
C_{1}(t)-C_{1}(0)=\frac{1}{\Gamma(\alpha)} \int_{0}^{t} P_{5}\left(\tau, C_{1}(\tau)\right)(t-\tau)^{\alpha-1} d \tau \\
C_{2}(t)-C_{2}(0)=\frac{1}{\Gamma(\alpha)} \int_{0}^{t} P_{6}\left(\tau, C_{2}(\tau)\right)(t-\tau)^{\alpha-1} d \tau
\end{gathered}
$$

Theorem 4. 1 In the region $\eta \times\left[t_{0}, T\right]$, where

$\eta=\left\{\left(C_{V}, C_{S D}, C_{L D}, C_{R}, C_{1}, C_{2}\right) \in \mathbb{R}^{6}: \max \left\{\left|\mathrm{C}_{\mathrm{V}}\right|,\left|\mathrm{C}_{\mathrm{SD}}\right|,\left|\mathrm{C}_{\mathrm{LD}}\right|,\left|\mathrm{C}_{\mathrm{R}}\right|,\left|\mathrm{C}_{1}\right|,\left|\mathrm{C}_{2}\right|\right\} \leq P\right\}$

and $T<+\infty$, the Lipschitz condition holds good and contraction occurs by the kernel if $P_{1}$ if $0 \leq \beta_{0}+\beta_{1} P<1$.

Proof: We consider the two functions $C_{V}(t)$ and $\bar{C}_{V}(t)$ such as:

$$
\begin{gathered}
\left\|\mathrm{P}_{1}\left(t, C_{V}\right)-\mathrm{P}_{1}\left(t, \bar{C}_{V}\right)\right\|=\left\|J_{V}-\beta_{0} C_{V}-\beta_{1} C_{1} C_{V}-J_{V}+\beta_{0} \bar{C}_{V}+\beta_{1} \bar{C}_{1} \bar{C}_{V}\right\| \\
\leq\left(\beta_{0}+\beta_{1} P\right)\left\|C_{V}(t)-\bar{C}_{V}(t)\right\| \\
\left\|\mathrm{P}_{1}\left(t, C_{V}\right)-\mathrm{P}_{1}\left(t, \bar{C}_{V}\right)\right\|=\chi_{1}\left\|C_{V}(t)-\bar{C}_{V}(t)\right\|
\end{gathered}
$$

where $\chi_{1}=\beta_{0}+\beta_{1} P$ implies that,

The Lipschitz condition is met for $P_{1}$ and if $0 \leq \chi_{1}<1$, then $P_{1}$ follows contraction. Similarly, it can be shown and illustrated in the other equations as follows:

$$
\begin{aligned}
& \left\|\mathrm{P}_{2}\left(t, C_{S D}\right)-\mathrm{P}_{2}\left(t, \bar{C}_{S D}\right)\right\| \leq \chi_{2}\left\|C_{S D}(t)-\bar{C}_{S D}(t)\right\| \\
& \left\|\mathrm{P}_{3}\left(t, C_{L D}\right)-\mathrm{P}_{3}\left(t, \bar{C}_{L D}\right)\right\| \leq \chi_{3}\left\|C_{L D}(t)-\bar{C}_{L D}(t)\right\| \\
& \left\|\mathrm{P}_{4}\left(t, C_{R}\right)-\mathrm{P}_{4}\left(t, \bar{C}_{R}\right)\right\| \leq \chi_{4}\left\|C_{R}(t)-\bar{C}_{R}(t)\right\| \\
& \left\|\mathrm{P}_{5}\left(t, C_{1}\right)-\mathrm{P}_{5}\left(t, \bar{C}_{1}\right)\right\| \leq \chi_{5}\left\|C_{1}(t)-\bar{C}_{1}(t)\right\| \\
& \left\|\mathrm{P}_{6}\left(t, C_{2}\right)-\mathrm{P}_{6}\left(t, \bar{C}_{2}\right)\right\| \leq \chi_{6}\left\|C_{2}(t)-\bar{C}_{2}(t)\right\|
\end{aligned}
$$

Where $\chi_{2}=\left(\delta_{S_{0}}+\delta_{S_{1}} P\right), \chi_{3}=\left(\delta_{L_{0}}+\delta_{L_{1}} P\right), \chi_{4}=\gamma_{0}, \chi_{5}=\left(\mu_{1}+\beta_{1} P\right)$ and $\chi_{6}=\mu_{2}+\left(\delta_{S_{1}}+\delta_{L_{1}}+\delta_{R}\right) P . P_{i}, i=2,3,4,5,6$ are the contraction if $0<\chi_{i}<1, i=2,3,4,5,6$. Using system 4 , the recursive form can now be written as:

$$
\begin{gathered}
\kappa_{1, n}(t)=C_{V_{n}}(t)-C_{V_{n-1}}(t)=\frac{1}{\Gamma(\alpha)} \int_{0}^{t}\left(P_{1}\left(\tau, C_{V_{n-1}}\right)-P_{1}\left(\tau, C_{V_{n-2}}\right)\right)(t-\tau)^{\alpha-1} d \tau \\
\kappa_{2, n}(t)=C_{S D_{n}}(t)-C_{S D_{n-1}}(t)=\frac{1}{\Gamma(\alpha)} \int_{0}^{t}\left(P_{2}\left(\tau, C_{S D_{n-1}}\right)-P_{2}\left(\tau, C_{S D_{n-2}}\right)\right)(t-\tau)^{\alpha-1} d \tau \\
\kappa_{3, n}(t)=C_{L D_{n}}(t)-C_{L D_{n-1}}(t)=\frac{1}{\Gamma(\alpha)} \int_{0}^{t}\left(P_{3}\left(\tau, C_{L D_{n-1}}\right)-P_{3}\left(\tau, C_{L D_{n-2}}\right)\right)(t-\tau)^{\alpha-1} d \tau \\
\kappa_{4, n}(t)=C_{R_{n}}(t)-C_{R_{n-1}}(t)=\frac{1}{\Gamma(\alpha)} \int_{0}^{t}\left(P_{4}\left(\tau, C_{R_{n-1}}\right)-P_{4}\left(\tau, C_{R_{n-2}}\right)\right)(t-\tau)^{\alpha-1} d \tau
\end{gathered}
$$




$$
\begin{gathered}
\kappa_{5, n}(t)=C_{1_{n}}(t)-C_{1_{n-1}}(t)=\frac{1}{\Gamma(\alpha)} \int_{0}^{t}\left(P_{5}\left(\tau, C_{1_{n-1}}\right)-P_{5}\left(\tau, C_{1_{n-2}}\right)\right)(t-\tau)^{\alpha-1} d \tau \\
\kappa_{6, n}(t)=C_{2_{n}}(t)-C_{2_{n-1}}(t)=\frac{1}{\Gamma(\alpha)} \int_{0}^{t}\left(P_{6}\left(\tau, C_{2_{n-1}}\right)-P_{6}\left(\tau, C_{2_{n-2}}\right)\right)(t-\tau)^{\alpha-1} d \tau
\end{gathered}
$$

The prerequisites are: $C_{V 0}(t)=C_{V}(0), C_{S D 0}(t)=C_{S D}(0), C_{L D 0}(t)=C_{L D}(0), C_{R 0}(t)=C_{R}(0), C_{10}(t)=C_{1}(0), C_{20}(t)=C_{2}(0)$. By applying the norm to the first equation 7 , we get

i

$$
\begin{aligned}
& \left\|\kappa_{1, n}(t)\right\|=\left\|C_{V_{n}}(t)-C_{V_{n-1}}(t)\right\|=\left\|\frac{1}{\Gamma(\alpha)} \int_{0}^{t}\left(P_{1}\left(\tau, C_{V_{n-1}}\right)-P_{1}\left(\tau, C_{V_{n-2}}\right)\right)(t-\tau)^{\alpha-1} d \tau\right\| \\
& \leq \frac{1}{\Gamma(\alpha)} \int_{0}^{t}\left\|\left(P_{1}\left(\tau, C_{V_{n-1}}\right)-P_{1}\left(\tau, C_{V_{n-2}}\right)\right)(t-\tau)^{\alpha-1} d \tau\right\|
\end{aligned}
$$

Using Lipchitz condition 5, we obtain

$$
\left\|\kappa_{1, n}(t)\right\| \leq \frac{1}{\Gamma(\alpha)} \chi_{1} \int_{0}^{t}\left\|\kappa_{1, n-1}(\tau) d \tau\right\|
$$

Similarly,

$$
\begin{aligned}
\left\|\kappa_{2, n}(t)\right\| & \leq \frac{1}{\Gamma(\alpha)} \chi_{2} \int_{0}^{t}\left\|\kappa_{2, n-1}(\tau) d \tau\right\| \\
\left\|\kappa_{3, n}(t)\right\| & \leq \frac{1}{\Gamma(\alpha)} \chi_{3} \int_{0}^{t}\left\|\kappa_{3, n-1}(\tau) d \tau\right\| \\
\left\|\kappa_{4, n}(t)\right\| & \leq \frac{1}{\Gamma(\alpha)} \chi_{4} \int_{0}^{t}\left\|\kappa_{4, n-1}(\tau) d \tau\right\| \\
\left\|\kappa_{5, n}(t)\right\| & \leq \frac{1}{\Gamma(\alpha)} \chi_{5} \int_{0}^{t}\left\|\kappa_{5, n-1}(\tau) d \tau\right\| \\
\left\|\kappa_{6, n}(t)\right\| & \leq \frac{1}{\Gamma(\alpha)} \chi_{6} \int_{0}^{t}\left\|\kappa_{6, n-1}(\tau) d \tau\right\|
\end{aligned}
$$

Now, it can be written as

$$
\begin{aligned}
& C_{V n}(t)=\sum_{i=1}^{n} \kappa_{1, i}, C_{S D n}(t)=\sum_{i=1}^{n} \kappa_{2, i}, C_{L D n}(t)=\sum_{i=1}^{n} \kappa_{3, i} . \\
& C_{R n}(t)=\sum_{i=1}^{n} \kappa_{4, i}, C_{1 n}(t)=\sum_{i=1}^{n} \kappa_{5, i}, C_{2 n}(t)=\sum_{i=1}^{n} \kappa_{6, i} .
\end{aligned}
$$

The above Theorem will be used to illustrate the proceeding Theorem.

Theorem 4. 2 The solution of the pluviculture model 2 exists and will be unique, if we acquire some $t_{\alpha}$ such that $\frac{1}{\Gamma(\alpha)} \chi_{i} t_{\alpha}<1$

for $\mathrm{i}=1,2,3,4,5,6$.

Proof: Applying equation 9 and 10 recursively, we have

$$
\begin{aligned}
& \left\|\kappa_{1, i}(t)\right\| \leq\left\|C_{V_{n}}\left(t_{0}\right)\right\|\left(\frac{1}{\Gamma(\alpha)} \chi_{1} \mathrm{t}\right)^{\mathrm{n}} \\
& \left\|\kappa_{2, i}(t)\right\| \leq\left\|C_{S D_{n}}\left(t_{0}\right)\right\|\left(\frac{1}{\Gamma(\alpha)} \chi_{2} \mathrm{t}\right)^{\mathrm{n}} \\
& \left\|\kappa_{3, i}(t)\right\| \leq\left\|C_{L D_{n}}\left(t_{0}\right)\right\|\left(\frac{1}{\Gamma(\alpha)} \chi_{3} \mathrm{t}\right)^{\mathrm{n}} \\
& \left\|\kappa_{4, i}(t)\right\| \leq\left\|C_{R_{n}}\left(t_{0}\right)\right\|\left(\frac{1}{\Gamma(\alpha)} \chi_{4} \mathrm{t}\right)^{\mathrm{n}} \\
& \left\|\kappa_{5, i}(t)\right\| \leq\left\|C_{1_{n}}\left(t_{0}\right)\right\|\left(\frac{1}{\Gamma(\alpha)} \chi_{5} \mathrm{t}\right)^{\mathrm{n}} \\
& \left\|\kappa_{6, i}(t)\right\| \leq\left\|C_{2_{n}}\left(t_{0}\right)\right\|\left(\frac{1}{\Gamma(\alpha)} \chi_{6} \mathrm{t}\right)^{\mathrm{n}}
\end{aligned}
$$


As a result, the existence and continuity are established. To illustrate that the relation 11 formulate the solution for 2, we assume the following:

$$
\begin{aligned}
& C_{V}(t)-C_{V}\left(t_{0}\right)=C_{V n}(t)-\varpi_{1 n}(t) \\
& C_{S D}(t)-C_{S D}\left(t_{0}\right)=C_{S D n}(t)-\varpi_{2 n}(t) \\
& C_{L D}(t)-C_{L D}\left(t_{0}\right)=C_{L D n}(t)-\varpi_{3 n}(t) \\
& C_{R}(t)-C_{R}\left(t_{0}\right)=C_{R n}(t)-\varpi_{4 n}(t) \\
& C_{1}(t)-C_{1}\left(t_{0}\right)=C_{1 n}(t)-\varpi_{5 n}(t) \\
& C_{2}(t)-C_{2}\left(t_{0}\right)=C_{2 n}(t)-\varpi_{6 n}(t)
\end{aligned}
$$

In order to achieve the desired outcomes, we set

$$
\left\|\varpi_{1 n}(t)\right\|=\left\|\frac{1}{\Gamma(\alpha)} \int_{0}^{t}\left(\mathrm{P}_{1}\left(\tau, C_{V}\right)-\mathrm{P}_{1}\left(\tau, C_{V_{n-1}}\right)\right) d \tau\right\|
$$

This yields

$$
\left\|\varpi_{1 n}(t)\right\| \leq \frac{1}{\Gamma(\alpha)} \chi_{1}\left\|C_{V}-C_{V_{n-1}}\right\| t
$$

Continuing the same procedure recursively, we get

$$
\left\|\varpi_{1 n}(t)\right\| \leq\left(\frac{1}{\Gamma(\alpha)} \chi_{1} \mathrm{t}\right)^{\mathrm{n}+1} P
$$

At $t_{\alpha}$, we have

$$
\left\|\varpi_{1 n}(t)\right\| \leq\left(\frac{1}{\Gamma(\alpha)} \chi_{1} \mathrm{t}\right)^{\mathrm{n}+1} P .
$$

From equation 13, it results that as $n$ tends to $\infty,\left\|\varpi_{1 n}(t)\right\|$ tends to 0 . In the same way, it may be demonstrated that $\left\|\varpi_{2 n}(t)\right\|,\left\|\varpi_{3 n}(t)\right\|,\left\|\varpi_{4 n}(t)\right\|,\left\|\varpi_{5 n}(t)\right\|,\left\|\varpi_{6 n}(t)\right\|$ tends to 0 . Hence the proof.

We will now demonstrate the uniqueness of the solution of the system 2 . Suppose that there is a different set of solution of the system 2 , namely $\widehat{C}_{V}, \widehat{C}_{S D}, \widehat{C}_{L D}, \widehat{C}_{R}, \widehat{C}_{1}, \widehat{C}_{2}$. Then, from the first equation of 4 we write

$C_{V}(t)-\widehat{C}_{V}(t)=\frac{1}{\Gamma(\alpha)} \int_{0}^{t}\left(P_{1}\left(t, C_{V}\right)-P_{1}\left(t, \widehat{C}_{V}\right)\right) d \tau$

Using the norm, the equation above becomes:

$$
\left\|C_{V}(t)-\hat{C}_{V}(t)\right\|=\frac{(1-\alpha)}{\Gamma(\alpha)} \int_{0}^{t}\left\|\left(\mathrm{P}_{1}\left(t, C_{V}\right)-\mathrm{P}_{1}\left(t, \hat{C}_{V}\right)\right) d \tau\right\|
$$

By applying the Lipschitz condition, we get

$$
\left\|C_{V}(t)-\hat{C}_{V}(t)\right\| \leq \frac{1}{\Gamma(\alpha)} \chi t\left\|C_{V}-\hat{C}_{V}\right\|
$$

This results in,

$$
\left\|C_{V}(t)-\hat{C}_{V}(t)\right\|\left(1-\frac{1}{\Gamma(\alpha)} \chi t\right) \leq 0
$$

Since $\left(1-\frac{1}{\Gamma(\alpha)} \chi_{1} t\right)>0$, we must have $\left\|C_{V}(t)-\hat{C}_{V}(t)\right\|=0$.. This implies $C_{V}(t)=\widehat{C}_{V}(t)$.

\section{Boundedness}

Here, we establish the boundedness of the solutions of the system 2.

Theorem 5. 1 The solutions of the pluviculture model 2 are uniformly bounded.

Proof. Let, $C(t)=C_{V}(t)+C_{S D}(t)+C_{L D}(t)+C_{R}(t)+C_{1}(t)+C_{2}(t)$

Taking the fractional Caputo derivative, we obtain

$$
\begin{aligned}
& { }_{t_{0}}^{C} D_{t}^{\alpha} C(t)+\mu_{1} C(t)={ }_{t_{0}}^{C} D_{t}^{\alpha}\left[C_{V}(t)+C_{S D}(t)+C_{L D}(t)+C_{R}(t)+C_{1}(t)+C_{2}(t)\right] \\
& +\mu_{1}\left[C_{V}(t)+C_{S D}(t)+C_{L D}(t)+C_{R}(t)+C_{1}(t)+C_{2}(t)\right] \\
& =J_{V}-\beta_{0} C_{V}-\beta_{1} C_{V} C_{1}+\delta_{S} C_{V}-\delta_{S_{0}} C_{S D}+\psi \beta_{1} C_{V} C_{1}-\delta_{S_{1}} C_{S D} C_{2} \quad(15) \\
& +\delta_{L} C_{S D}-\delta_{L_{0}} C_{L D}+\delta_{S_{1}} C_{S D} C_{2}-\delta_{L_{1}} C_{L D} C_{2}+\gamma C_{L D}-\gamma_{0} C_{R}+\xi \delta_{L_{1}} C_{L D} C_{2} \\
& +J_{1}-\mu_{1} C_{1}-\beta_{1} C_{V} C_{1}+J_{2}-\mu_{2} C_{2}-\delta_{S_{1}} C_{S D} C_{2}-\delta_{L_{1}} C_{L D} C_{2}-\delta_{R} C_{R} C_{2} \\
& +\mu_{1}\left[C_{V}(t)+C_{S D}(t)+C_{L D}(t)+C_{R}(t)+C_{1}(t)+C_{2}(t)\right] \\
& \leq J_{V}+\delta_{S} C_{V}+\psi \beta_{1} C_{V} C_{1}+\delta_{L} C_{S D}+\gamma C_{L D}+\xi \delta_{L_{1}} C_{L D} C_{2} \\
& +J_{1}+J_{2}+\mu_{1}\left[C_{V}(t)+C_{S D}(t)+C_{L D}(t)+C_{R}(t)+C_{2}(t)\right]
\end{aligned}
$$


The solution exists and is unique in

$$
\eta=\left\{\left(C_{V}, C_{S D}, C_{L D}, C_{R}, C_{1}, C_{2}\right): \max \left\{\left(C_{V} \mid,\left(C_{S D} \mid,\left(C_{L D} \mid,\left(C_{R} \mid,\left(C_{1} \mid,\left(C_{2} \mid\right\} \leq P\right\}\right.\right.\right.\right.\right.\right.
$$

The above inequality yields,

${ }_{t_{0}}^{C} D_{t}^{\alpha} C(t)+\mu_{1} C(t) \leq J_{V}+J_{1}+J_{2}+P\left(\delta_{S}+\psi \beta_{1} P+\delta_{L}+\gamma+\xi \delta_{L_{1}}+5 \mu_{1}\right)$

By the lemma 2, we get

$$
{ }_{t_{0}}^{C} D_{t}^{\alpha} C(t) \leq\left(C\left(t_{0}\right)-\frac{1}{\mu_{1}}\left(J_{V}+J_{1}+J_{2}+P\left(\delta_{S}+\psi \beta_{1} P+\delta_{L}+\gamma+\xi \delta_{L_{1}}+5 \mu_{1}\right)\right)\right) E_{\alpha}\left(-\theta\left(t-t_{0}\right)^{\alpha}\right)
$$

$+\frac{1}{\mu_{1}}\left(J_{V}+J_{1}+J_{2}+P\left(\delta_{S}+\psi \beta_{1} P+\delta_{L}+\gamma+\xi \delta_{L_{1}}+5 \mu_{1}\right)\right) \rightarrow J_{V}+J_{1}+J_{2}+P\left(\delta_{S}+\psi \beta_{1} P+\delta_{L}+\gamma+\xi \delta_{L_{1}}+5 \mu_{1}\right), t \rightarrow \infty$

Therefore, all the solution of the system 2 that initiates in $\eta$ remained bounded in

$$
\Xi=\left(\left(C_{V}, C_{S D}, C_{L D}, C_{R}, C_{1}, C_{2}\right) \in \eta \mid C(t) \leq J_{V}+J_{1}+J_{2}+P\left(\delta_{S}+\psi \beta_{1} P+\delta_{L}+\gamma+\xi \delta_{L_{1}}+5 \mu_{1}\right)+\varepsilon, \varepsilon>0\right\}
$$

\section{Existence of the Points of Equilibrium and the Stability}

The system 2 has the following set of interesting points of equilibrium. The criteria for the stability of the set of equilibrium points have been discussed here. The Jacobian matrix of the pluviculture model 2 is

$$
J\left(C_{V}, C_{S D}, C_{L D}, C_{R}, C_{1}, C_{2}\right)=\left(\begin{array}{cccccc}
J_{11} & J_{12} & J_{13} & J_{14} & J_{15} & J_{16} \\
J_{21} & J_{22} & J_{23} & J_{24} & J_{25} & J_{26} \\
J_{31} & J_{32} & J_{33} & J_{34} & J_{35} & J_{36} \\
J_{41} & J_{42} & J_{43} & J_{44} & J_{45} & J_{46} \\
J_{51} & J_{52} & J_{53} & J_{54} & J_{55} & J_{56} \\
J_{61} & J_{62} & J_{63} & J_{64} & J_{65} & J_{66}
\end{array}\right)
$$

where

$$
\begin{gathered}
J_{11}=-\beta_{0}-C_{1} \beta_{1}, \quad J_{12}=0, \quad J_{13}=0, \quad J_{14}=0, \quad J_{15}=C_{V} \beta_{1}, \quad J_{16}=0, \\
J_{21}=C_{1} \psi \beta_{1}+\delta_{S}, \quad J_{22}=-\delta_{S_{0}}-C_{2} \delta_{S_{1}}, \quad J_{23}=0, \quad J_{24}=0, \quad J_{25}=C_{V} \psi \beta_{1}, \quad J_{26}=-C_{S D} \delta_{S_{1}}, \\
J_{31}=0, \quad J_{32}=\delta_{L}+C_{2} \delta_{S_{1}}, \quad J_{33}=-\delta_{L_{0}}-C_{2} \delta_{L_{1}}, \quad J_{34}=0, \quad J_{35}=0, \quad J_{36}=-C_{L D} \delta_{L_{1}}+C_{S D} \delta_{S_{1}}, \\
J_{41}=0, \quad J_{42}=0, \quad J_{43}=\gamma+C_{2} \xi \delta_{L_{1}}, \quad J_{44}=-\gamma_{0}, \quad J_{45}=0, \quad J_{46}=C_{L D} \xi \delta_{L_{1}}, \\
J_{51}=-C_{1} \beta_{1}, \quad J_{52}=0, \quad J_{53}=0, \quad J_{54}=0, \quad J_{55}=-C_{V} \beta_{1}-\mu_{1}, \quad J_{56}=0, \\
J_{61}=0, \quad J_{62}=-C_{2} \delta_{S_{1}}, \quad J_{63}=-C_{2} \delta_{L_{1}}, \quad J_{64}=-C_{2} \delta_{R}, \quad J_{65}=0,
\end{gathered}
$$

$J_{66}=-C_{L D} \delta_{L_{1}}-C_{R} \delta_{R}-C_{S D} \delta_{S_{1}}-\mu_{2}$.

Equilibrium points of the pluviculture model 2 is discussed below:

1. Axial equilibrium point is $\mathfrak{E}=\left(\frac{J_{V}}{\beta_{0}}, 0,0,0,0,0\right)$. 
Theorem 6.1 Axial equilibrium point $E$ always exists and is always stable.

Proof. At $E$, Jacobian matrix $J$ of system 2 has the eigenvalues:

$$
\lambda_{11}=-\beta_{0}, \quad \lambda_{12}=-\delta_{L_{0}}, \quad \lambda_{13}=-\delta_{S_{0}}, \quad \lambda_{14}=-\gamma_{0}, \quad \lambda_{15}=-\left(\frac{J_{V} \beta_{1}+\beta_{0} \mu_{1}}{\beta_{0}}\right), \quad \lambda_{16}=-\mu_{2} .
$$

Since all the above eigen values are negative, the axial equilibrium point $E$ is always stable.

2. Aerosol free equilibrium point is \widetilde $\widetilde{\mathfrak{E}}=\left(\tilde{C}_{V}, \tilde{C}_{S D}, \tilde{C}_{L D}, \tilde{C}_{R}, 0,0\right)$

Theorem $\underset{\widetilde{E}}{6.2}$ Aerosol free equilibrium point $\widetilde{E}$ always exists and is always stable.

Proof. At $\widetilde{E}$, Jacobian matrix $J$ of system 2 has the eigenvalues:

$$
\begin{gathered}
\lambda_{11}=-\beta_{0}, \quad \lambda_{12}=-\delta_{L_{0}}, \quad \lambda_{13}=-\delta_{S_{0}}, \quad \lambda_{14}=-\gamma_{0}, \quad \lambda_{15}=-\left(\frac{J_{V} \beta_{1}+\beta_{0} \mu_{1}}{\beta_{0}}\right) \\
\lambda_{16}=-\left(\frac{J_{V} \delta_{S}\left(\gamma \delta_{L} \delta_{R}+\gamma_{0}\left(\delta_{L} \delta_{L_{1}}+\delta_{L_{0}} \delta_{S_{1}}\right)\right)+\beta_{0} \delta_{L_{0}} \delta_{S_{0}} \gamma_{0} \mu_{2}}{\beta_{0} \delta_{L_{0}} \delta_{S_{0}} \gamma_{0}}\right) .
\end{gathered}
$$

Since all the above eigen values are negative, the aerosol free equilibrium point $\widetilde{E}$ is always stable.

\section{Numerical Method}

In the present section, we have presented the generalized Adams-Bashforth-Moulton technique ${ }^{(32)}$ to solve the pluviculture model 2 .

Consider,

${ }^{C} D_{t}^{\alpha} x(t)=\phi(t, x(t)), \quad 0 \leq t \leq T x^{(m)}(0)=x_{0}^{(m)}, \quad m=0,1,2,3, \ldots ., v \ldots, v=\lceil\alpha\rceil$.

The corresponding Volterra integral equation may be written as

$$
x(t)=\sum_{m=0}^{v-1} x_{0}^{(m)} \frac{t^{m}}{m !}+\frac{1}{\Gamma(\alpha)} \int_{0}^{t}(t-s)^{\alpha-1} \phi(s, x(s)) d s .
$$

In order to integrate 16, Adams-Bashforth Moultan method has been used by Diethelm et al. ${ }^{(33,34)}$. Set $h=\frac{T}{N}, t_{n}=n h$, $n=0,1,2, \ldots, N \in Z$. Now, the system 2 can be written as:

$$
\begin{aligned}
& C_{V n+1}=C_{V 0}+\frac{h^{\alpha}}{\Gamma(\alpha+2)}\left[J_{V}-\beta_{0} C_{V}^{p}{ }_{n+1}^{p}-\beta_{1} C_{V}^{p}{ }_{n+1}^{p} C_{1}^{p}{ }_{n+1}\right] \\
& +\frac{h^{\alpha}}{\Gamma(\alpha+2)} \sum_{i=0}^{n} a_{i, n+1}\left[J_{V}-\beta_{0} C_{V i}-\beta_{1} C_{V i} C_{1 i}\right] \text {, } \\
& C_{S D n+1}=C_{S D 0}+\frac{h^{\alpha}}{\Gamma(\alpha+2)}\left[\delta_{S} C_{V}^{p}{ }_{n+1}-\delta_{S_{0}} C_{S D_{n+1}^{p}}^{p}+\psi \beta_{1} C_{V}^{p}{ }_{n+1} C_{1}^{p}{ }_{n+1}^{p}-\delta_{S_{1}} C_{S D_{n+1}^{p}}^{p} C_{2}^{p}\right] \\
& +\frac{h^{\alpha}}{\Gamma(\alpha+2)} \sum_{i=0}^{n} a_{i, n+1}\left[\delta_{S} C_{V i}-\delta_{S_{0}} C_{S D i}+\psi \beta_{1} C_{V i} C_{1 i}-\delta_{S_{1}} C_{S D i} C_{2 i}\right] \text {, } \\
& C_{L D n+1}=C_{L D 0}+\frac{h^{\alpha}}{\Gamma(\alpha+2)}\left[\delta_{L} C_{S D_{n+1}^{p}}^{p}-\delta_{L_{0}} C_{L D_{n+1}^{p}}^{p}+\delta_{S_{1}} C_{S D_{n+1}^{p}}^{p} C_{2}^{p}{ }_{n+1}^{p}-\delta_{L_{1}} C_{L D_{n+1}^{p}}^{p} C_{2 n+1}^{p}\right] \\
& +\frac{h^{\alpha}}{\Gamma(\alpha+2)} \sum_{i=0}^{n} a_{i, n+1}\left[\delta_{L} C_{S D i}-\delta_{L_{0}} C_{L D i}+\delta_{S_{1}} C_{S D i} C_{2 i}-\delta_{L_{1}} C_{L D i} C_{2 i}\right] \text {, } \\
& C_{R n+1}=C_{R 0}+\frac{h^{\alpha}}{\Gamma(\alpha+2)}\left[\gamma C_{L D_{n+1}^{p}}^{p}-\gamma_{0} C_{R}^{p}+\xi \delta_{L_{1}} C_{L D_{n+1}}^{p} C_{2}^{p}{ }_{n+1}^{p}\right] \\
& +\frac{h^{\alpha}}{\Gamma(\alpha+2)} \sum_{i=0}^{n} a_{i, n+1}\left[\gamma C_{L D i}-\gamma_{0} C_{R i}+\xi \delta_{L_{1}} C_{L D i} C_{2 i}\right] \\
& C_{1 n+1}=C_{10}+\frac{h^{\alpha}}{\Gamma(\alpha+2)}\left[J_{1}-\mu_{1} C_{1_{n+1}}^{p}-\beta_{1} C_{V}^{p}{ }_{n+1}^{p} C_{1_{n+1}^{p}}^{p}\right] \\
& +\frac{h^{\alpha}}{\Gamma(\alpha+2)} \sum_{i=0}^{n} a_{i, n+1}\left[J_{1}-\mu_{1} C_{1 i}-\beta_{1} C_{V i} C_{1 i}\right] \text {, } \\
& C_{2 n+1}=C_{20}+\frac{h^{\alpha}}{\Gamma(\alpha+2)}\left[J_{2}-\mu_{2} C_{2 n+1}^{p}-\delta_{S_{1}} C_{S D_{n+1}^{p}}^{p} C_{2}^{p}{ }_{n+1}^{p}-\delta_{L_{1}} C_{L D_{n+1}^{p}}^{p} C_{2 n+1}^{p}-\delta_{R} C_{R}^{p}{ }_{n+1} C_{2}^{p}{ }_{n+1}^{p}\right] \\
& +\frac{h^{\alpha}}{\Gamma(\alpha+2)} \sum_{i=0}^{n} a_{i, n+1}\left[J_{2}-\mu_{2} C_{2 i}-\delta_{S_{1}} C_{S D i} C_{2 i}-\delta_{L_{1}} C_{L D i} C_{2 i}-\delta_{R} C_{R i} C_{2 i}\right],
\end{aligned}
$$


where

$$
\begin{aligned}
& C_{V_{n+1}^{p}}^{p}=C_{V_{0}}+\frac{h^{\alpha}}{\Gamma(\alpha+1)} \sum_{i=0}^{n} b_{i, n+1}\left[J_{V}-\beta_{0} C_{V_{i}}-\beta_{1} C_{V_{i}} C_{1_{i}}\right] \\
& C_{S D_{n+1}^{p}}=C_{S D_{0}}+\frac{h^{\alpha}}{\Gamma(\alpha+1)} \sum_{i=0}^{n} b_{i, n+1}\left[\delta_{S} C_{V_{i}}-\delta_{S_{0}} C_{S D_{i}}+\psi \beta_{1} C_{V_{i}} C_{1_{i}}-\delta_{S_{1}} C_{S D_{i}} C_{2_{i}}\right] \\
& C_{L D_{n+1}^{p}}=C_{L D_{0}}+\frac{h^{\alpha}}{\Gamma(\alpha+1)} \sum_{i=0}^{n} b_{i, n+1}\left[\delta_{L} C_{S D_{i}}-\delta_{L_{0}} C_{L D_{i}}+\delta_{S_{1}} C_{S D_{i}} C_{2_{i}}-\delta_{L_{1}} C_{L D_{i}} C_{2_{i}}\right] \\
& C_{R_{n+1}^{p}}=C_{R_{0}}+\frac{h^{\alpha}}{\Gamma(\alpha+1)} \sum_{i=0}^{n} b_{i, n+1}\left[\gamma C_{L D_{i}}-\gamma_{0} C_{R_{i}}+\xi \delta_{L_{1}} C_{L D_{i}} C_{2 i}\right] \\
& C_{1}^{p} n_{n+1}=C_{1_{0}}+\frac{h^{\alpha}}{\Gamma(\alpha+1)} \sum_{i=0}^{n} b_{i, n+1}\left[J_{1}-\mu_{1} C_{1_{i}}-\beta_{1} C_{V_{i}} C_{1_{i}}\right] \\
& C_{2_{n+1}^{p}}^{p}=C_{2_{0}}+\frac{h^{\alpha}}{\Gamma(\alpha+1)} \sum_{i=0}^{n} b_{i, n+1}\left[J_{2}-\mu_{2} C_{2_{i}}-\delta_{S_{1}} C_{S D_{i}} C_{2_{i}}-\delta_{L_{1}} C_{L D_{i}} C_{2_{i}}-\delta_{R} C_{R_{i}} C_{2_{i}}\right]
\end{aligned}
$$

in which

$$
a_{i, n+1}=\left\{\begin{array}{cl}
n^{\alpha_{j}+1}-\left(n-\alpha_{j}\right)(n+1)^{\alpha_{j}}, & i=0, \\
(n-i+2)^{\alpha_{j}+1}+(n-i)^{\alpha_{j}+1}-2(n-i+1)^{\alpha_{j}+1}, & 1 \leq i \leq n \\
1, & i=n+1
\end{array}\right.
$$

and

$$
b_{i, n+1}=\frac{h^{\alpha_{j}}}{\alpha_{j}}\left((n-i+1)^{\alpha_{j}}-(n-i)^{\alpha_{j}}\right), 0 \leq i \leq n,
$$

with $j=1,2,3,4,5,6$.

\section{Numerical Simulation}

In order to discuss the dynamics of the projected fractional pluviculture model 2, we have used the generalized AdamsBashforth-Moulton technique by considering the parametric values as $J_{V} \in(0,3), \beta_{0}=1, \beta_{1}=0.5, \delta_{S}=0.1, \delta_{S_{0}}=1, \psi=$ $1, \delta_{S_{1}}=0 ., \delta_{L}=0.1, \delta_{L_{0}}=1, \delta_{L_{1}}=0.3, \gamma=0.1, \gamma_{0}=0.02, \xi=1, J_{1} \in(0,3), J_{2} \in(0,3), \mu_{1}=1, \mu_{2}=1$ and $\delta_{R}=0.01$ with the initial conditions $C_{V}=0.7545, C_{S D}=0.2422, C_{L D}=0.0862, C_{R}=0.4919, C_{1}=0.6507$ and $C_{2}=0.8534$. For $J_{V}=0,1,2,3$, we can observe the variation in $C_{V}, C_{S D}, C_{L D}$ and $C_{R}$ in Figures 1, 2 and 3 for $\alpha=1,0.8,0.5$ respectively. As the natural formation of water vapor $\left(J_{V}\right)$ increases, $C_{V}, C_{S D}, C_{L D}$ and $C_{R}$ also increase. As the fractional value are incorporated, the increasing trend is retained whereas the net value slightly decrease.

For $J_{1}=0,1,2,3$, we can observe the variation in $C_{V}, C_{S D}, C_{L D}$ and $C_{R}$ in Figures 4,5 and 6 for $\alpha=1,0.8,0.5$ respectively. As the rate of entry of first type of aerosols $\left(J_{1}\right)$ into the environment increases, the value of $C_{V}$ starts decreasing since natural formation of water vapors will be less than that of due to first kind aerosol. Whereas, the values of $C_{S D}, C_{L D}$ and $C_{R}$ shows the increasing trend and hence the rainfall is stimulated by this action.

For $J_{2}=0,1,2,3$, we can observe the variation in $C_{S D}, C_{L D}$ and $C_{R}$ in Figures 7,8 and 9 for $\alpha=1,0.8,0.5$ respectively. As the rate of entry of second type of aerosols $\left(J_{2}\right)$ into the environment increases, the value of $C_{S D}$ starts decreasing since the conversion of small drops into large drops and then to rain drops is more than that of the small drops formation. Hence the values of $C_{L D}$ and $C_{R}$ is increased and induces more rainfall in the environment than the previous.

Figures 10, 11 and 12 depicts the global stability of $C_{1}-C_{V}, C_{2}-C_{S D}$ and $C_{2}-C_{L D}$ space for $\alpha=1,0.8$ respectively. Solution paths of system 2 starting in the domain of attraction are attaining equilibrium values herein.

\section{Results and Discussion}

By considering the previous results and values as that of in ${ }^{(15)}$ along with other predicted values for the new parameters used in our present model, the dynamics of the model is studied by using generalized Adams-Bashforth-Moulton technique. In the above reference, only one kind of aerosol is used whereas, we have proposed our model by the aid of two kinds of aerosols 


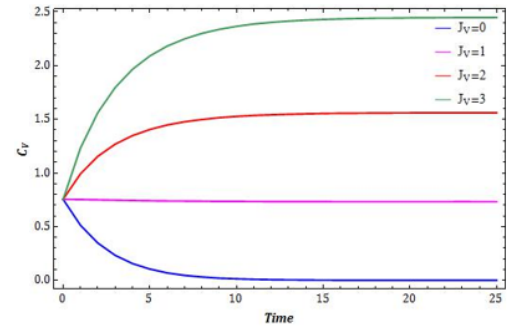

(A)

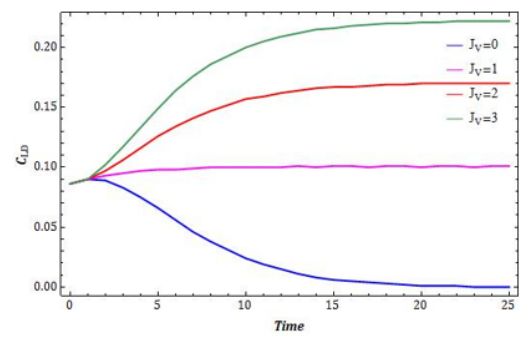

(C)

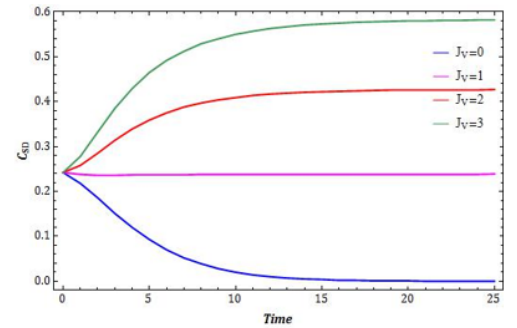

(B)

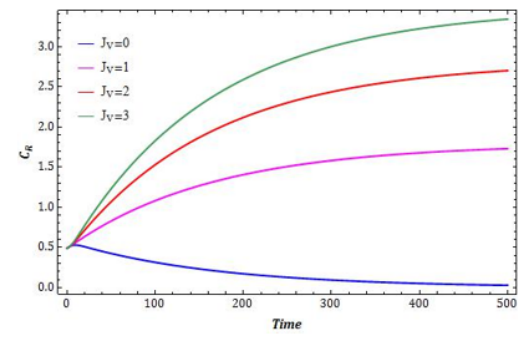

(D)

Fig 1. Variation in the (A) $C_{V}$, (B) $C_{S D}$, (C) $C_{L D}$ and (D) $C_{R}$ for different values of $J_{V}$ for $\alpha=1$

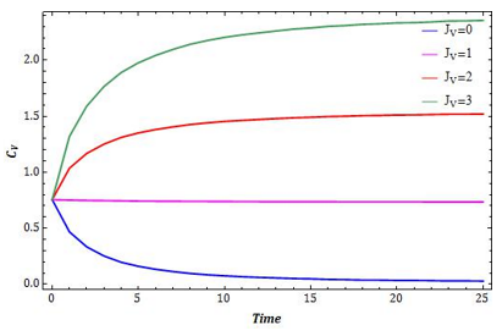

(A)

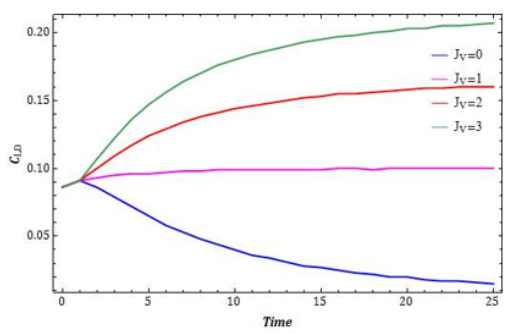

(C)

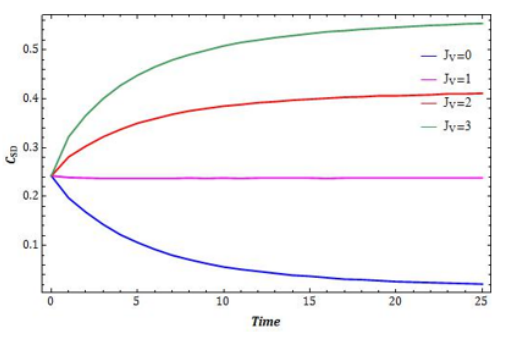

(B)

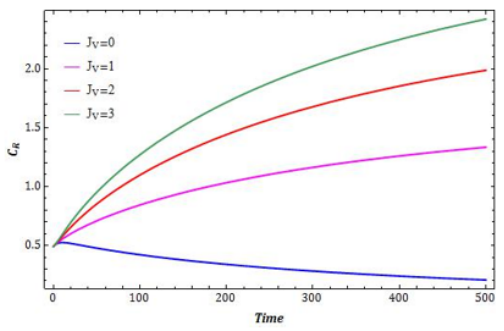

(D)

Fig 2. Variation in the (A) $C_{V}$, (B) $C_{S D}$, (C) $C_{L D}$ and (D) $C_{R}$ for different values of $J_{V}$ for $\alpha=0.8$ 


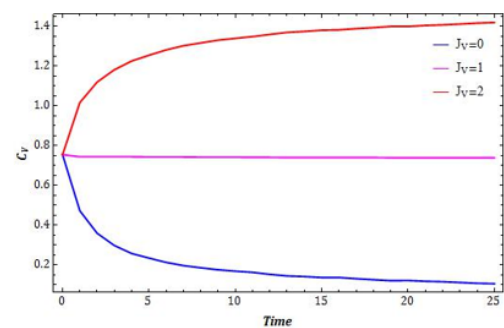

(A)

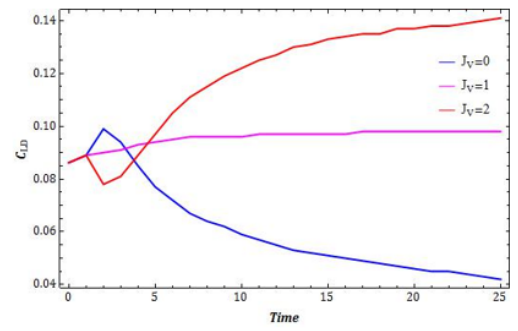

(C)

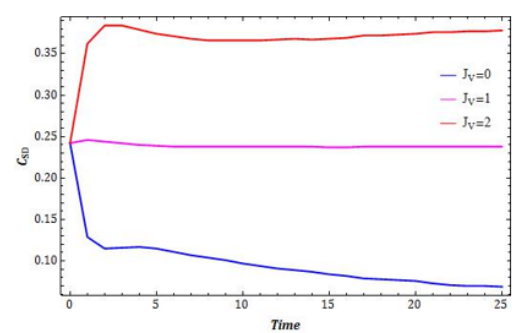

(B)

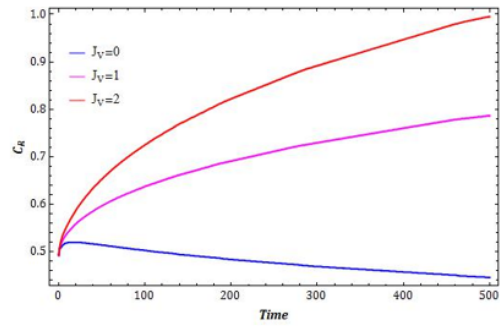

(D)

Fig 3. Variation in the (A) $C_{V}$, (B) $C_{S D}$, (C) $C_{L D}$ and (D) $C_{R}$ for different values of $J_{V}$ for $\alpha=0.5$

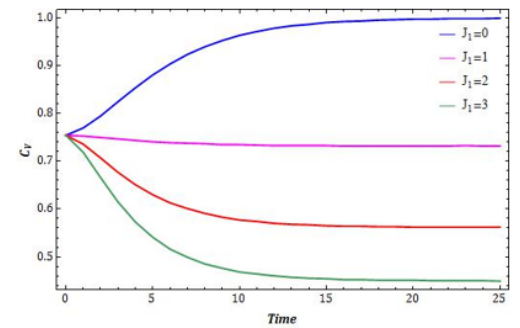

(A)

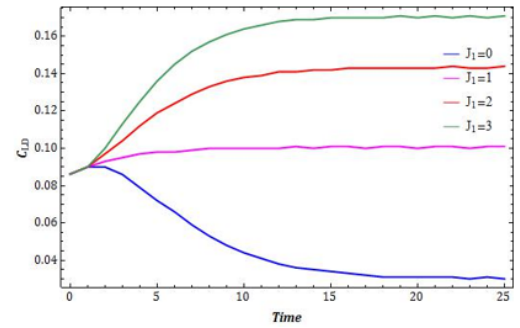

(C)

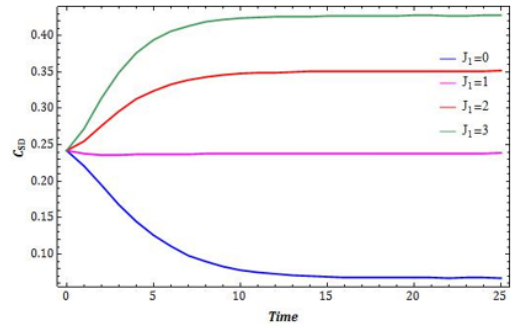

(B)

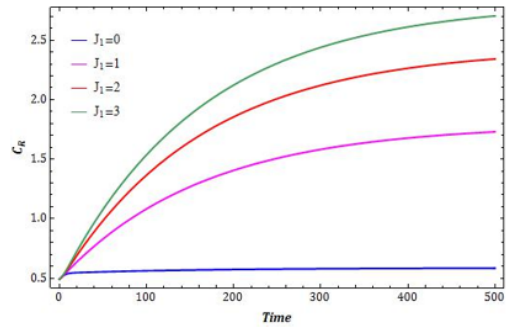

(D)

Fig 4. Variation in the (A) $C_{V}$, (B) $C_{S D}$, (C) $C_{L D}$ and (D) $C_{R}$ for different values of $J_{1}$ for $\alpha=1$ 


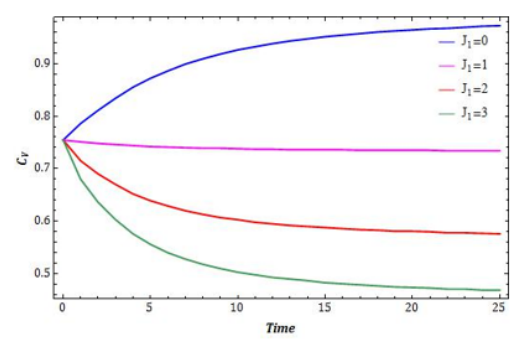

(A)

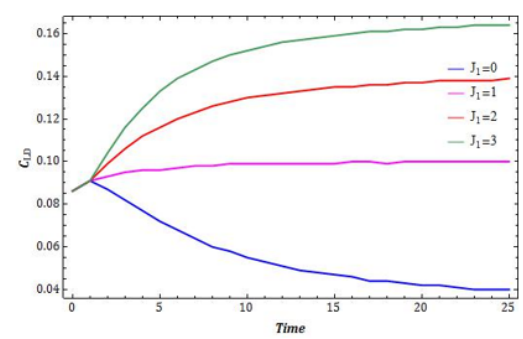

(C)

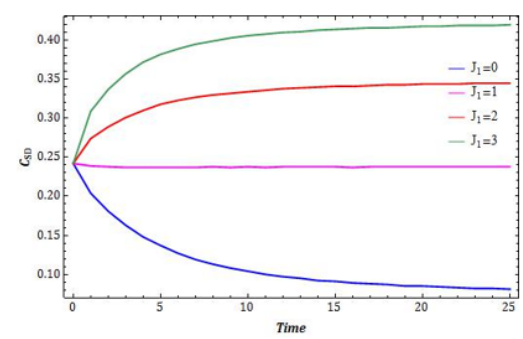

(B)

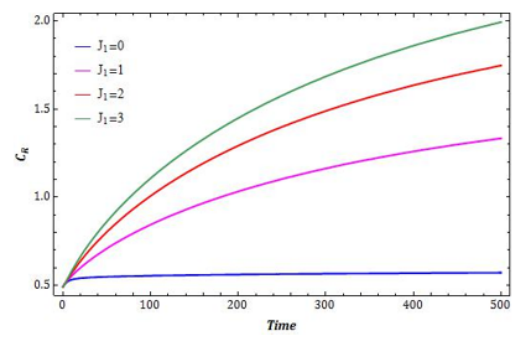

(D)

Fig 5. Variation in the (A) $C_{V}$, (B) $C_{S D}$, (C) $C_{L D}$ and (D) $C_{R}$ for different values of $J_{1}$ for $\alpha=0.8$

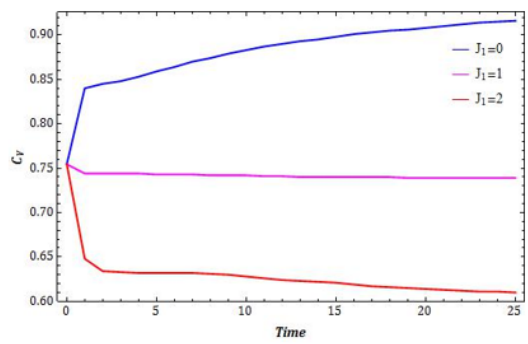

(A)

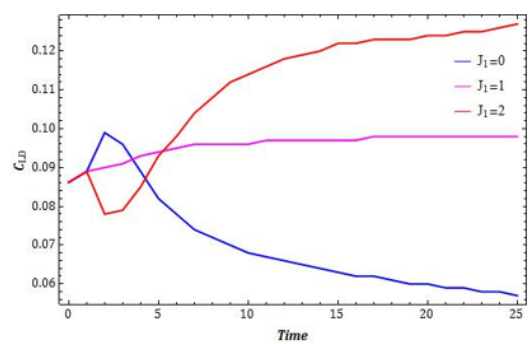

(C)

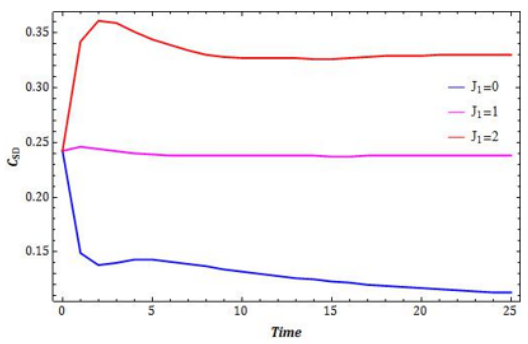

(B)

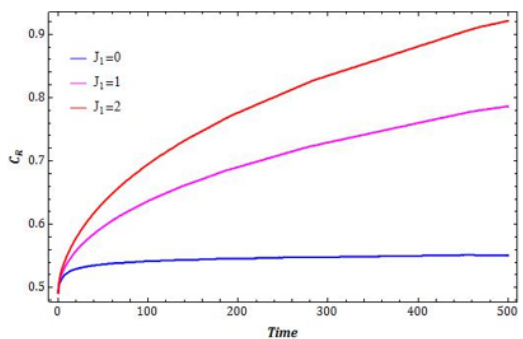

(D)

Fig 6. Variation in the (A) $C_{V}$, (B) $C_{S D}$, (C) $C_{L D}$ and (D) $C_{R}$ for different values of $J_{1}$ for $\alpha=0.5$. 


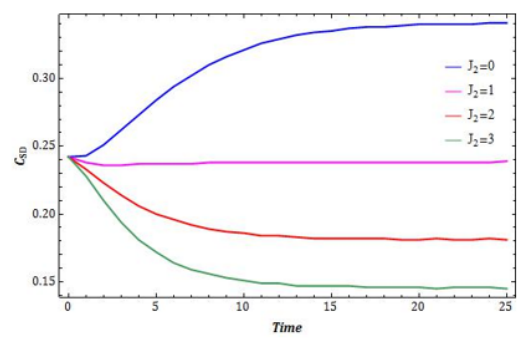

(A)

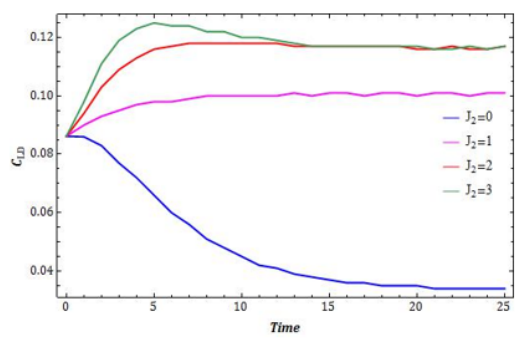

(B)

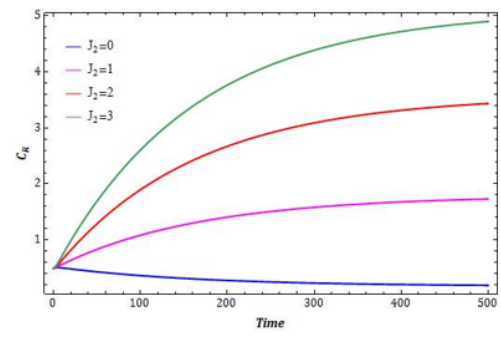

(C)

Fig 7. Variation in the (A) $C_{S D}$, (B) $C_{L D}$ and (C) $C_{R}$ for different values of $J_{2}$ for $\alpha=1$.

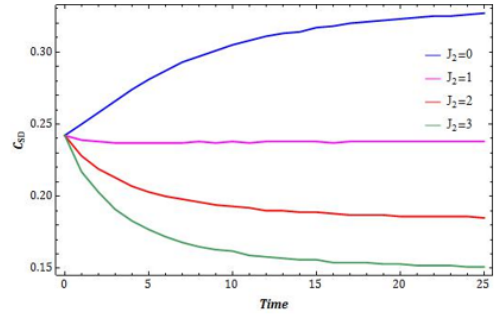

(A)

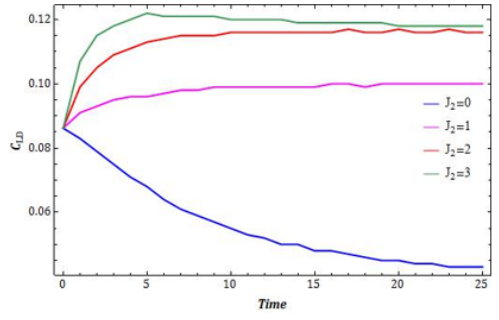

(B)

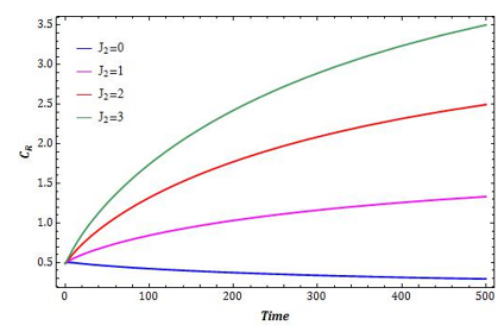

(C)

Fig 8. Variation in the (A) $C_{S D}$, (B) $C_{L D}$ and (C) $C_{R}$ for different values of $J_{2}$ for $\alpha=0.8$ 


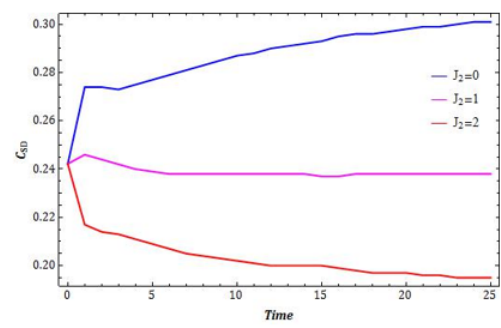

(A)

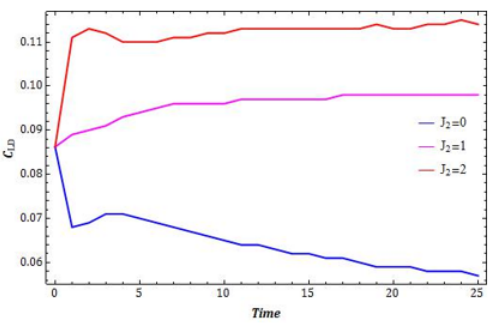

(B)

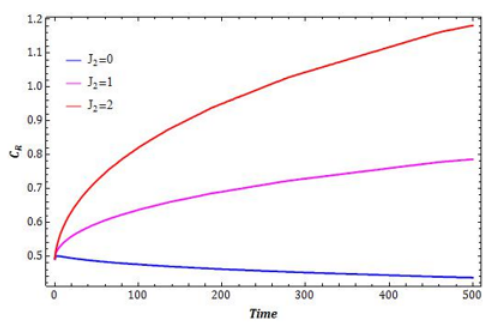

(C)

Fig 9. Variation in the (A) $C_{S D}$, (B) $C_{L D}$ and (C) $C_{R}$ for different values of $J_{2}$ for $\alpha=0.5$

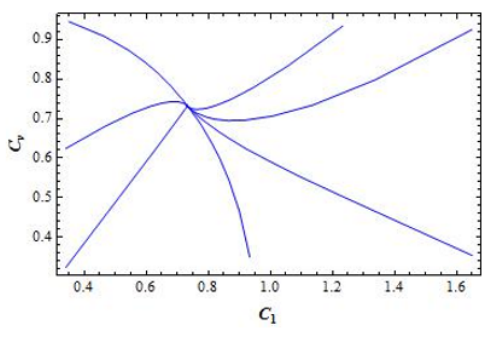

(A)

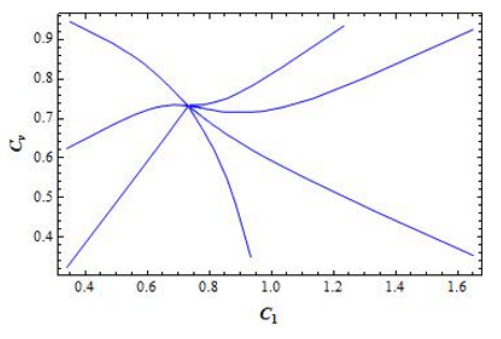

(B)

Fig 10. Global stability in $C_{1}-C_{V}$ space for (A) $\alpha=1$ and (B) $\alpha=0.8$.

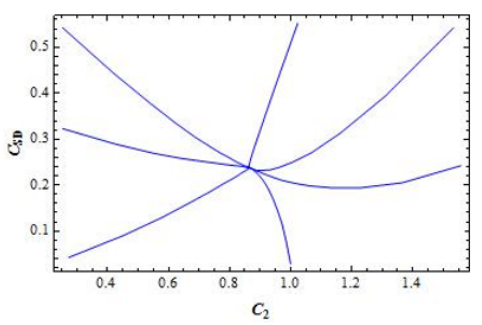

(A)

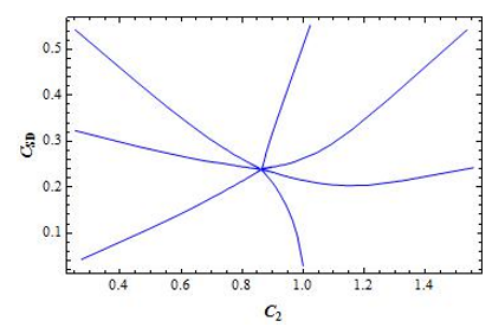

(B)

Fig 11. Global stability in $C_{2}-C_{S D}$ space for (A) $\alpha=1$ and (B) $\alpha=0.8$. 


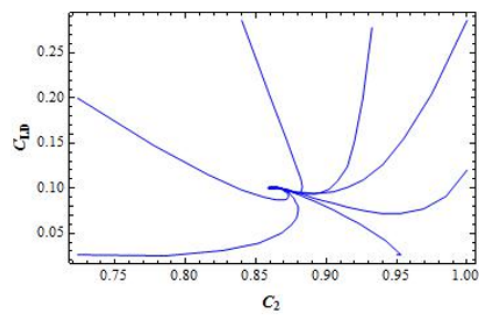

(A)

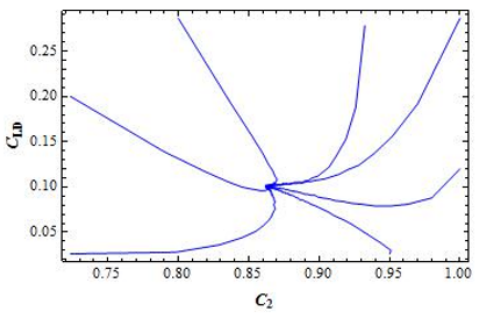

(B)

Fig 12. Global stability in $C_{2}-C_{L D}$ space for (A) $\alpha=1$ and (B) $\alpha=0.8$.

along with the Caputo fractional derivative. We see that, the results are in good agreement with the previous ones and also the results are more realistic when the fractional values are incorporated which is discussed in the Section (8). We have also analyzed the global stability numerically for different fractional values which is not done in the previous works so far. Overall, the pluviculture model incorporating the fractional derivatives is the novel, and we see the effect of different parameters with different fractional values which gives the better and realistic representation of our proposed model.

\section{Conclusion}

A non-linear mathematical approach for pluviculture by introducing particles into the atmosphere is suggested and analyzed in this study. The effect of fractional order derivative is numerically analyzed and it is established that numerical simulation strengthens the analytical results of the model. According to the model, rainfall occurs only when the water vapor are concentrated on the nature, not on a continual basis. In order for water vapor molecules to develop in the atmosphere, they must be continually generated. According to the study, rainfall is enhanced when the accumulative concentration of favorable aerosol particles rises. Pluviculture model with the fractional derivatives along with the two kinds of aerosols is the novel of the model which provides the more realistic representation of the model. The results obtained are more convincing and holds good with the real world phenomena that can be predicted.

\section{References}

1) Tripathi A, Misra AK, Shukla JB. A mathematical model for the removal of pollutants from the atmosphere through artificial rain. Stochastic Analysis and Applications. 2021;1(20):1-20. Available from: https://dx.doi.org/10.1080/07362994.2021.1915802.

2) Misra AK, Tripathi A. A stochastic model for making artificial rain using aerosols. Physica A: Statistical Mechanics and its Applications. 2018;505:11131126. Available from: https://dx.doi.org/10.1016/j.physa.2018.04.054.

3) Shafi M, Jha RK, Sabraj M. A survey on security issues of 5G NR: Perspective of artificial dust and artificial rain. Journal of Network and Computer Applications. 2020;160:102597-102597. Available from: https://dx.doi.org/10.1016/j.jnca.2020.102597.

4) Misra AK, Tripathi A. An optimal control model for cloud seeding in a deterministic and stochastic environment. Optimal Control Applications and Methods. 2020;41(6):2166-2189. Available from: https://dx.doi.org/10.1002/oca.2648.

5) Mcgrath K. Artificial rain: Control, precision and reality in Anthony Chen's wet season. Metro Magazine: Media e Education Magazine. 2021 ;p. 80-85. Available from: https://search.informit.org/doi/abs/10.3316/informit.034577127091537.

6) Yue Z, Shansuo Z, Xiaohang LIU. Constitutive model of confined concrete with stirrups by acid rain erosion. IOP Conference Series: Earth and Environmental Science. 2021;643(1):012026-012026. Available from: https://dx.doi.org/10.1088/1755-1315/643/1/012026.

7) Yu Y, Hopkins C. Empirical models for the structure-borne sound power input from artificial and natural rainfall. Applied Acoustics. 2020;162:107199107199. Available from: https://dx.doi.org/10.1016/j.apacoust.2019.107199.

8) Zubaidi SL, Abdulkareem IH, Hashim KS, Al-Bugharbee H, Ridha HM, Gharghan SK, et al. Hybridised Artificial Neural Network Model with Slime Mould Algorithm: A Novel Methodology for Prediction of Urban Stochastic Water Demand. Water. 2020;12(10):2692-2692. Available from: https://dx.doi.org/10.3390/w12102692.

9) Jagodnik V, Peranić J, Željko Arbanas. Mechanism of Landslide Initiation in Small-Scale Sandy Slope Triggered by an Artificial Rain. Understanding and Reducing Landslide Disaster Risk. 2021;p. 177-184. Available from: https://doi.org/10.1007/978-3-030-60713-5_19.

10) Verma P, Chakraborty S. Now casting of Orographic Rain Rate Using ARIMA and ANN Model. 2020 URSI Regional Conference on Radio Science (URSIRCRS). 2020;p. 1-4. Available from: https://doi.org/10.23919/URSIRCRS49211.2020.9113563.

11) Anwar MT, Hadikurniawati W, Winarno E, Widiyatmoko W. Perfomance Comparison of Data Mining Techniques for Rain Prediction Models in Indonesia. In: and others, editor. 2020 3rd International Seminar on Research of Information Technology and Intelligent Systems (ISRITI). 2020;p. 83-88. Available from: https://doi.org/10.1109/ISRITI51436.2020.9315460.

12) Arbanas Z, Jagodnik V, Peranic J, Pajalic S, Prodan MV, Ceh N. Physical model of rainfall induced landslide in flume test: preliminary results. 2020.

13) Diba FD, Samad MA, Choi DY. The Effects of Rain on Terrestrial Links at K, Ka and E-Bands in South Korea: Based on Supervised Learning. IEEE Access. 2021;9:9345-9355. Available from: https://dx.doi.org/10.1109/access.2021.3049825. 
14) Lata K, Misra AK. The influence of forestry resources on rainfall: A deterministic and stochastic model. Applied Mathematical Modelling. 2020;81:673-689. Available from: https://dx.doi.org/10.1016/j.apm.2020.01.009.

15) Misra AK, Tripathi A, Naresh R, Shukla JB. Modelling and analysis of the effects of aerosols in making artificial rain. Modeling Earth Systems and Environment. 2016;2:1-11. Available from: https://dx.doi.org/10.1007/s40808-016-0228-1.

16) Podlubny I. Fractional differential equations: An introduction to fractional derivatives, fractional differential equations, to methods of their solution and some of their applications. . 1998.

17) Losada J, Nieto JJ. Properties of a new fractional derivative without singular kernel. Progr Fract Differ Appl. 2015;1(2):87-92. Available from: http://dx.doi.org/10.12785/pfda/010202.

18) Dokuyucu MA, Celik E, Bulut H, Baskonus HM. Cancer treatment model with the Caputo-Fabrizio fractional derivative. The European Physical Journal Plus. 2018;133(3):1-6. Available from: https://dx.doi.org/10.1140/epjp/i2018-11950-y.

19) Khan MA, Hammouch Z, Baleanu D. Modeling the dynamics of hepatitis E via the Caputo-Fabrizio derivative. Mathematical Modelling of Natural Phenomena. 2019;14(3):311-311. Available from: https://dx.doi.org/10.1051/mmnp/2018074.

20) Baleanu D, Jajarmi A, Mohammadi H, Rezapour S. A new study on the mathematical modelling of human liver with Caputo-Fabrizio fractional derivative. Chaos, Solitons \& Fractals. 2020;134:109705-109705. Available from: https://dx.doi.org/10.1016/j.chaos.2020.109705.

21) Ghanbari B, Günerhan H, Srivastava HM. An application of the Atangana-Baleanu fractional derivative in mathematical biology: A three-species predatorprey model. Chaos, Solitons \& Fractals. 2020;138:109910-109910. Available from: https://dx.doi.org/10.1016/j.chaos.2020.109910.

22) Shah SAA, Khan MA, Farooq M, Ullah S, Alzahrani EO. A fractional order model for Hepatitis B virus with treatment via Atangana-Baleanu derivative. Physica A: Statistical Mechanics and its Applications. 2020;538:122636-122636. Available from: https://dx.doi.org/10.1016/j.physa.2019.122636.

23) Owolabi KM. Analysis and numerical simulation of multicomponent system with Atangana-Baleanu fractional derivative. Chaos, Solitons \& Fractals. 2018;115:127-134. Available from: https://doi.org/10.1016/j.chaos.2018.08.022.

24) Huang C, Li H, Cao J. A novel strategy of bifurcation control for a delayed fractional predator-prey model. Applied Mathematics and Computation. 2019;347:808-838. Available from: https://dx.doi.org/10.1016/j.amc.2018.11.031.

25) Ghanbari B, Kumar D. Numerical solution of predator-prey model with Beddington-DeAngelis functional response and fractional derivatives with MittagLeffler kernel. Chaos: An Interdisciplinary Journal of Nonlinear Science. 2019;29(6):063103-063103. Available from: https://dx.doi.org/10.1063/1.5094546.

26) Baishya C. Dynamics of fractional stage structured predator prey model with prey refuge. Indian Journal of Ecology. 2020;47(4):1118-1124. Available from: https://doi.org/10.1063/1.5094546.

27) Baishya C. Dynamics of Fractional Holling Type-II Predator-Prey Model with Prey Refuge and Additional Food to Predator. Journal of Applied Nonlinear Dynamics. 2021;10(2):315-328. Available from: https://dx.doi.org/10.5890/jand.2021.06.010.

28) Li HL, Zhang L, Hu C, Jiang YL, Teng Z. Dynamical analysis of a fractional-order predator-prey model incorporating a prey refuge. Journal of Applied Mathematics and Computing. 2017;54(1-2):435-449. Available from: https://dx.doi.org/10.1007/s12190-016-1017-8.

29) Baishya C, Achar SJ, Veeresha P, Prakasha DG. Dynamics of a fractional epidemiological model with disease infection in both the populations. Chaos: An Interdisciplinary Journal of Nonlinear Science. 2021;31(4):043130-043130. Available from: https://dx.doi.org/10.1063/5.0028905.

30) Wang B, Chen LQ. Asymptotic stability analysis with numerical confirmation of an axially accelerating beam constituted by the standard linear solid model. Journal of Sound and Vibration. 2009;328(4-5):456-466. Available from: https://dx.doi.org/10.1016/j.jsv.2009.08.016.

31) Li C, Tao C. On the fractional Adams method. Computers \& Mathematics with Applications. 2009;58(8):1573-1588. Available from: https://dx.doi.org/ 10.1016/j.camwa.2009.07.050.

32) Diethelm K. An algorithm for the numerical solution of differential equations of fractional order. Electronic transactions on numerical analysis. 1997;5(1):16. Available from: https://doi.org/10.1.1.48.5509.

33) Diethelm K, Ford NJ. Analysis of Fractional Differential Equations. Journal of Mathematical Analysis and Applications. 2002;265(2):229-248. Available from: https://dx.doi.org/10.1006/jmaa.2000.7194.

34) Diethelm K, Ford NJ, Freed AD. A predictor-corrector approach for the numerical solution of fractional differential equations. Nonlinear Dynamics. 2002;29(1):3-22. Available from: https://doi.org/10.1023/A:1016592219341. 